\section{Systemmykosen bei Leukämiekranken: Caspofungin breit wirksam, gut verträglich und interaktionsarm}

Eine häufige - möglicherweise inzwischen sogar die maßgebliche - Ursache für den Tod von Leukämiekranken nach hochdosierter Chemotherapie oder Stammzelltransplantation sind invasive Pilzinfektionen. Mit Caspofungin steht jetzt ein innovatives Antimykotikum zur Verfügung, das sowohl dem gewandelten Erregerspektrum als auch der Komorbidität und Komedikation der immungeschwächten Patienten Rechnung trägt.

Seit den 1980er Jahren wird eine stete Zunahme von nosokomialen Systemmykosen beobachtet. Bei malignen hämatologischen Erkrankungen ist dies nach Aussage von Prof. Noel Milpied, Nantes/Frankreich, im Wesentlichen darauf zurückzuführen, dass die $\mathrm{Pa}$ tienten zum einen auch bei schlechtem Allgemeinzustand immer «immunotoxischeren» Therapien ausgesetzt werden und dass zum anderen die bakteriell- und viralbedingte Letalität stark rückläufig ist. In großen Autopsiestudien habe sich zum Zeitpunkt des Todes bei mindestens jedem fünften Fall von Leukämie/Knochenmarktransplantation eine invasive Pilzinfektion nachweisen lassen.

Doch nicht nur die Inzidenz der Systemmykosen hat zugenommen, sondern gleichzeitig auch ihre Pathogenität. Bei den Hefepilzen sind Candida albicans und Candida tropicalis rückläufig zu Gunsten von - schwerer eradizierbaren - Spezies wie Candida krusei oder Candida glabrata. Ebenfalls auf dem Vormarsch sind Schimmelpilze. Als Beispiel nannte Milpied eine Statistik des Krebszentrums an der Universität Seattle/USA. Bei Patienten mit allogener Stamm- zelltransplantation gab es dort zwischen 1990 und 1998 bei den Aspergillosen einen Anstieg von etwa 5 auf $12 \%$ pro Jahr. Eine Zunahme wurde bei den seltenen Infektionen durch Fusari- und Zygomyceten beobachtet [1].

\section{Caspofungin erweitert das antimykotische Repertoire}

Erfreulicherweise zugenommen hat auch die Auswahl an therapeutischen Alternativen. Referenzsubstanz ist aufgrund seines breiten Wirkspektrums nach wie vor Amphotericin B. Die liposomale Variante bringt diesbezüglich keinen weiteren Vorteil. Milpied wies darauf hin, dass jedes weniger breit wirksame Antimykotikum den potentiellen Nachteil habe, die Verbreitung der nicht erfassten Spezies zu begünstigen. Die bei knochenmarktransplantierten Patienten beobachtete Verschiebung von Albicans- zu Non-albicans-Candidosen bzw. von Hefe- zu Schimmelpilzinfektionen sei parallel gegangen mit der vielfach üblichen prophylaktischen Anwendung von Fluconazol [2]. Ein ähnlicher Trend, nämlich eine Zunahme von Zygomykosen, lasse sich jetzt nach Einführung von Voriconazol beobachten [3].

Vor dem Hintergrund der möglichen therapie- oder altersbedingten Funktionseinschränkungen von Niere und Leber und der in der Regel erforderlichen Polymedikation darf man nach Dafürhalten von Milpied bei der Wahl des Antimykotikums für onkologische Patienten auch Faktoren wie Organto- xizität und Arzneimittelinteraktionen nicht aus den Augen verlieren. In dieser Hinsicht Pluspunkte mit einem darüber hinaus bemerkenswerten therapeutischen Index weist für ihn Caspofungin (CANCIDAS ${ }^{\circledR}$ ) auf. Die derzeitige Zulassung umfasst den Einsatz bei

- empirischer Therapie (siehe folgende Seite),

- invasiven Candidosen ohne Neutropenie,

- invasiven Aspergillosen bei Refraktärität oder Intoleranz gegenüber Standardantimykotika

In den Anfang 2004 aktualisierten IDSALeitlinien (Infectious Disases Society of America) wird Caspofungin - gleichberechtigt mit den verschiedenen Formulierungen von Amphotericin B und Fluconazol bzw. deren Kombination - zur First-line-Therapie nachgewiesener invasiver Candidosen empfohlen [4]

Caspofungin ist ein Echinocandin. Dabei handelt es sich um eine ganz neue Substanzklasse, die keinerlei Kreuzresistenzen mit Polyenen und Azolen aufweist. Der fungizide Effekt beruht auf der Hemmung der Beta-(1,3)-Glucan-Synthase, einem Enzymsystem, das nur in der Membran von Pilz-, nicht aber von Säugetierzellen vorhanden ist. Das erkläre, so Dr. Rodrigo Martino, Barcelona/Spanien, warum nahezu keine Organtoxizität zu erwarten ist - in seinen Augen ein extrem wichtiger Gesichtspunkt gerade für Patienten mit Leukämie und Stammzelltransplantation [5] (Abb. 1). Die antimykotische Aktivität decke das gesamte Spektrum invasiver Candida- und Aspergillusspezies und darüber hinaus eine Reihe weiterer pathogener Pilze ab.

Die Handhabung ist unkompliziert. Aufgrund der langen Halbwertszeit muss Caspofungin nur einmal täglich über eine Stunde infundiert werden - am ersten Tag $70 \mathrm{mg}$, danach jeweils $50 \mathrm{mg}$. Bei Niereninsuffizienz ist keine Dosisanpassung erforderlich, bei Leberfunktionsstörungen sollte nach initialer Aufsättigung die Dosis halbiert werden. Ein weiterer Vorteil von Caspofungin ist für Martino das geringe Interaktionspotential. Das gelte auch für Ciclosporin. Die in frühen Studien bei gesunden Probanden beobachteten Leberwerterhöhungen bei gleichzeitiger Gabe von Caspofungin mit dem Standardimmunsuppressivum hätten sich, so Martino, im klinischen Alltag als nicht relevant erwiesen. In neueren Untersuchungen seien keine unerwarteten Neben- oder Wechselwirkungen beobachtet worden.

\section{Stellenwert unter den Bedingungen des klinischen Alltags bestätigt}

Die zuverlässige Wirksamkeit von Caspofungin bei invasiven Candidosen ist inzwischen durch zahlreiche Untersuchungen belegt. Als Beispiel hob Martino die Ergeb-

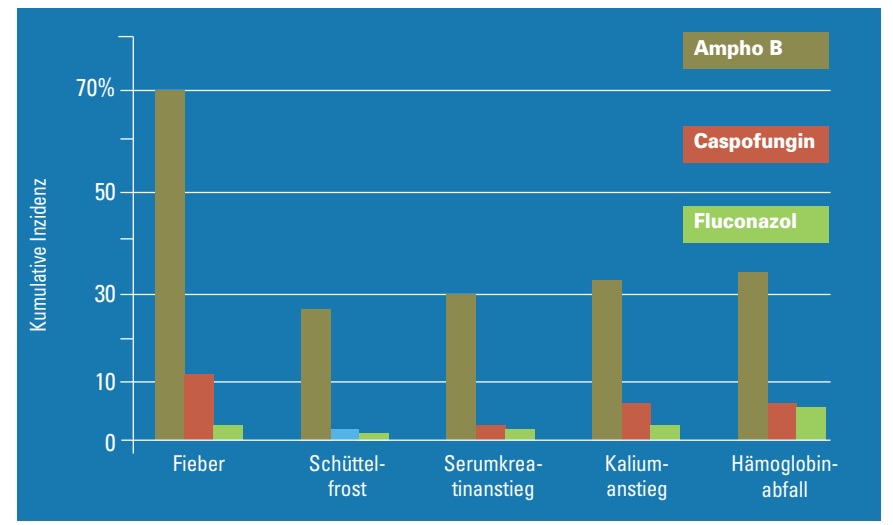




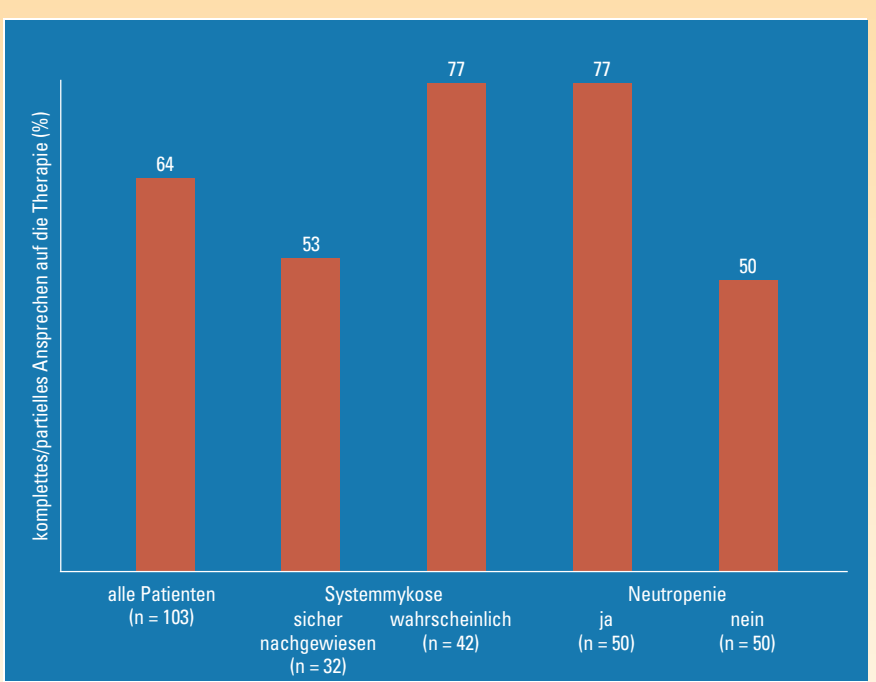

Abb. 2. Komplette oder partielle Remission von Systemmykose nach im Median 16-tägiger Therapie mit Caspofungin (nach Glasmacher, 2004).

nisse des doppelblinden Vergleichs mit Amphotericin $\mathrm{B}$ hervor [6]. Beim primären Endpunkt (keine Infektionssymptome / kein Erregernachweis am Ende der i.v. Therapie) war Caspofungin in der MITT-Analyse (Modified Intention-To-Treat $=$ mindestens eine Dosis des Antimykotikums / n = 224) im Trend wirksamer als die Standardmedikation $(73$ versus $62 \% / p=0,09)$ und sogar statistisch signifikant überlegen (81 versus $65 \% / \mathrm{p}=0,03$ ) bei dem Kollektiv, das mindestens 10 Tage antimykotisch behandelt worden war $(n=185)$. Die höhere Erfolgschance beim Einsatz von Caspofungin galt auch für Patienten mit Non-albicans-Candidosen ( 80 versus $68 \%$ ), Neutropenie (50 versus $40 \%$ ) oder Blutkrebs (61 versus $50 \%$ ).

Als etabliert bezeichnete Martino die Second-line-Therapie mit Caspofungin bei systemischen Aspergillosen, wenn die Patienten auf die antimykotische Standardbehandlung nicht ansprechen oder sie nicht vertragen. Dabei könne man sich auf die Ergebnisse von drei Studien mit Erfolgsraten zwischen 44 und 54\% stützen [7-9].

$\mathrm{Ob}$ die in kontrollierten Studien bei selektierten Patientenpopulationen dokumentierte Wirksamkeit von Caspofungin sich auch unter den heterogenen Bedingungen des klinischen Alltags bestätigt, wurde kürzlich in Deutschland untersucht. Für die Auswertung standen Daten von 118 Patienten mit Systemmykosen zur Verfügung. Beim Einschluss hatte es keine Restriktionen in Hinblick auf Art oder Schwere der Grunderkrankung bzw. Vor- oder Komedikation gegeben. In der Mehrzahl der Fälle waren es Patienten mit Leukämie (35\%), allogener/autologer Stammzelltransplantation (26\%) oder Lymphomen (14\%). Die Hälfte der Patienten war neutropenisch und ein Viertel musste maschinell beatmet werden. Obwohl es sich um eine Hochrisikogruppe gehandelt habe, seien unter Caspofungin keine schweren Nebenwirkungen aufgetre- ten, bestätigte PD Dr. Axel Glasmacher, Bonn, die aus den kontrollierten Studien bekannte gute Verträglichkeit. Auch bei den biochemischen Markern für die Nierenbzw- Leberfunktion habe man keine relevanten Veränderungen gesehen - unabhängig davon, ob eine Basismedikation mit $\mathrm{Ci}$ closporin $(n=14)$ bestanden habe oder nicht.

Auch die Erfolgsraten waren mindestens ebenso gut wie in den kontrollierten Studien (Abb. 2). In 65\% der Fälle wurde eine komplette oder partielle (keine klinischen/mikrobiellen Mykosezeichen, aber radiologische Residuen) Remission dokumentiert. Dies ist nach Ansicht von Glasmacher umso beachtlicher, als $77 \%$ der Patienten mit einem oder mehreren Antimykotika vorbehandelt gewesen seien.

\section{Empirischer Einsatz von Caspofungin}

Die Crux bei Systemmykosen ist ihre unspezifische Symptomatik und das Fehlen von Routinemethoden zum Erregernachweis im frühen Stadium der Infektion. Bei persistierendem Fieber trotz Therapie mit einem Breitspektrumantibiotikum bleibt nur der empirische Einsatz eines Antimykotikums. Die bis vor kurzem einzigen zugelassenen Optionen haben nach Aussage von Prof. Georg Maschmeyer, Berlin, jedoch alle ihre Limitationen - konventionellem/ liposomalem Amphotericin B mangele es an Verträglichkeit und Fluconazol an breiter Wirksamkeit. Voriconazol sei die Zulassung zum Einsatz als empirische Therapie von der amerikanischen Arzneimittelbehörde FDA verweigert worden.

Ein viel versprechender Kandidat aufgrund seines günstigen Nutzen-Risiko-Profils ist für Maschmeyer Caspofungin. Seine Einschätzung beruht auf dem Ausgang der bisher größten prospektiven Studie, die jemals zur empirischen Therapie bei Patienten mit febriler Neutropenie, die nicht auf die First-line-Therapie mit einem Breitspektrum-Antibiotikum angesprochen hatten $(n=1111)$, durchgeführt wurde. Ursache der Abwehrschwäche war in $94 \%$ eine maligne hämatologische Erkrankung/Stammzelltransplantation. Im doppelblinden Vergleich erwies sich Caspofungin als ebenso wirksam wie liposomales Amphotericin B, jedoch als statistisch signifikant überlegen bei Verträglichkeitsparametern wie Inzidenz substanz-/infusionsassoziierter Nebenwirkungen oder Nephrotoxizität $(p<0,001)$ [10]. Seit Mai 2004 ist Caspofungin auch zur empirischen Therapie - bei Verdacht auf Infektionen durch Pilze wie Candida- und Aspergillus-Arten - bei erwachsenen Patienten mit Fieber und Neutropenie zugelassen.

\section{Literatur}

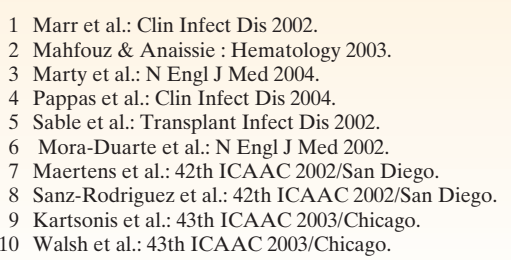

\section{Quelle}

Satellitensymposium «Important New Evidence to Overcome the Burden of Fungal Infections with Caspofungin in BMT Patients» beim 30th Annual Meeting of the European Group for Blood and Marrow Transplantation», 28. März 2004, Barcelona/ Spanien.

Bericht: Gabriele Blaeser-Kiel, Hamburg

\section{Impressum}

Systemmykosen bei Leukämiekranken: Caspofungin breit wirksam, gut verträglich und interaktionsarm

\section{PharmaForum in Onkologie 27 | 3 | 04}

(c) 2004 by S. Karger Verlag für Medizin und Naturwissenschaften $\mathrm{GmbH}$

Lörracher Straße 16a

D-79115 Freiburg

Tel. +49761452070

E-mail Information@Karger.de

Mit freundlicher Unterstützung der MSD SHARP und DOHME GMBH.

Der Verlag und die Herausgeber der Zeitschrift übernehmen keine Verantwortung für diese Rubrik.

Die Wiedergabe von Gebrauchsnamen, Handelsnamen, Warenbezeichnungen usw. in dieser Zeitschrift berechtigt auch ohne besondere Kennzeichnung nicht zur Annahme, dass solche Namen im Sinne der Warenzeichen- und Markenschutz-Gesetzgebung als frei zu betrachten wären und daher von jedermann benutzt werden dürfen. Für Angaben von Dosierungsanweisungen und Applikationsformen kann vom Verlag keine Haftung übernommen werden. Derartige Angaben müssen vom jeweiligen Anwender im Einzelfall anhand anderer Literaturstellen auf ihre Richtigkeit überprüft werden. 